\title{
The requirement for surgery and subsequent 30-day mortality in patients with COVID-19
}

\author{
Blayne Welk, MD, MSc \\ Lucie Richard, MSc \\ Sebastian Rodriguez-Elizalde, \\ MD, MHSc
}

Accepted February 4, 2021

\author{
Correspondence to: \\ B. Welk \\ Department of Surgery and Epidemiology \\ \& Biostatistics \\ Western University \\ Room B4-667 \\ St Joseph's Health Care \\ 268 Grosvenor St \\ London ON N6A 4V2 \\ bkwelk@gmail.com
}

DOI: $10.1503 /$ cjs. 022020

\section{SUMmaRY}

The ongoing COVID-19 pandemic has had profound effects on the provision of surgical care. The potential perioperative mortality associated with surgical procedures in patients with COVID-19 has been estimated at $20 \%$, but the data come from jurisdictions that experienced very high surges of COVID-19 patients. A rapid assessment of the types of surgical care for patients with COVID-19 in Ontario was carried out using administrative data, and we found that during the initial wave in the spring of 2020 , surgical interventions were required in $0.6 \%$ of patients with COVID-19, and mortality was higher $(20 \%)$ in patients who underwent surgery in the 2 weeks before or after a positive nasopharygeal swab than in those who had surgery more than 2 weeks after COVID-19 was diagnosed.

he COVID-19 pandemic led to unprecedented changes to surgical care. The increased risk of death while providing necessary surgical care to patients in the time shortly before and after a diagnosis of COVID-19 is an evolving area of study. ${ }^{1,2}$ Although there is an obvious preference to avoid surgery in patients with COVID-19, some emergency surgeries cannot be delayed. As the pandemic continues and cases increase in Canada, we felt it would be important to describe the surgical care and mortality of patients with COVID-19 from a Canadian perspective.

We analyzed multiple linked, routinely collected data sources from the universal health care system of Ontario, Canada, (population 14.7 million) through ICES. We used data from the Ontario Health Insurance Plan, Ontario Laboratory Information System, the Discharge Abstract Database/ Same Day Care Database, and the Registered Persons Database. Our review included all Ontario adults who had a positive nasopharyngeal swab for SARSCoV-2 between Feb. 1, 2020, and May 31, 2020. We identified the subgroup of patients who had a surgical procedure (determined by relevant surgeon and anesthesiologist billing codes and evidence of hospital admission). We excluded surgeries likely related to COVID-19 severity (e.g., tracheostomy) and endoscopies. Procedures were reported on an individual level, and only data on the first surgical procedure were included. Comorbidities relevant to COVID-19 were measured. The primary outcome was postsurgical 30-day mortality, and rates from the early and late period after COVID-19 diagnosis were compared using the Fisher exact test. In keeping with privacy regulations, no groups with fewer than 5 patients are identified, and numbers are masked where appropriate to prevent recalculation of these numbers. The use of data in this project was authorized under Ontario's Personal Health Information Protection Act, which does not require review by a Research Ethics Board. Patient consent was not required.

We identified 24322 people with COVID-19, and within this group 146 $(0.6 \%)$ had a relevant surgical procedure. Patients who underwent a surgical procedure were older and more likely to have comorbidities relevant to COVID-19 (Table 1). The provision of operative procedures was greatest 
Table 1. Demographic and surgical characteristics of patients with COVID-19 who did and did not undergo surgery

\begin{tabular}{|c|c|c|c|}
\hline Characteristic & $\begin{array}{c}\text { Nonsurgical group } \\
n=24176\end{array}$ & $\begin{array}{l}\text { Surgical group } \\
\quad n=146\end{array}$ & $\begin{array}{l}\text { Standardized } \\
\text { difference* }\end{array}$ \\
\hline Age, median (IQR) & 55 (39-73) & 65 (44-79) & 0.27 \\
\hline Female, $n(\%)$ & $10471(43.3 \%)$ & $61(41.8 \%)$ & 0.03 \\
\hline In long-term care, $n(\%)$ & 4820 (19.9\%) & $28(19.2 \%)$ & 0.02 \\
\hline \multicolumn{4}{|c|}{ Month of COVID-19 diagnosis, $n(\%)$} \\
\hline February & $14(0.1 \%)$ & $0(0.0 \%)$ & 0.03 \\
\hline March & $3,012(12.5 \%)$ & $16(11.0 \%)$ & 0.05 \\
\hline April & $12686(52.5 \%)$ & $84(57.5 \%)$ & 0.10 \\
\hline May & $8,464(35.0 \%)$ & $46(31.5 \%)$ & 0.07 \\
\hline \multicolumn{4}{|l|}{ Comorbidities, $n(\%) \dagger$} \\
\hline COPD & 877 (3.6\%) & $12(8.2 \%)$ & 0.20 \\
\hline Diabetes & $4,260(17.6 \%)$ & $31(21.2 \%)$ & 0.09 \\
\hline Heart failure & $1,184(4.9 \%)$ & $14(9.6 \%)$ & 0.18 \\
\hline Hypertension & $5,426(22.4 \%)$ & $48(32.9 \%)$ & 0.23 \\
\hline Heart disease & $679(2.8 \%)$ & $9(6.2 \%)$ & 0.16 \\
\hline Stroke/TIA & $1,075(4.4 \%)$ & $8(5.5 \%)$ & 0.05 \\
\hline Obesity (BMI > 40) & $1,000(4.1 \%)$ & $10(6.8 \%)$ & 0.12 \\
\hline Chronic kidney disease & $828(3.4 \%)$ & $14(9.6 \%)$ & 0.25 \\
\hline Cancer & $954(3.9 \%)$ & $16(11.0 \%)$ & 0.27 \\
\hline \multicolumn{4}{|l|}{ ASA classification } \\
\hline 1 or 2 & NA & $17(11.6 \%)$ & - \\
\hline 3 & NA & $40(27.4 \%)$ & - \\
\hline 4 & NA & $76(52.1 \%)$ & - \\
\hline 5 & NA & $13(8.9 \%)$ & - \\
\hline Emergency surgical procedure & NA & $98(67.1 \%)$ & - \\
\hline \multicolumn{4}{|l|}{ ICU admission } \\
\hline $1-3$ days before surgery & NA & $7(4.8 \%)$ & - \\
\hline $0-3$ days after surgery & NA & $14(9.6 \%)$ & - \\
\hline \multicolumn{4}{|c|}{$\begin{array}{l}\text { ASA = American Society of Anesthesiologists; BMI = body mass index; } C O P D=\text { chronic obstructive pulmonary } \\
\text { disease; ICU = intensive care unit; IQR = interquartile range; } T I A=\text { Transient Ischemic Attack. }\end{array}$} \\
\hline \multicolumn{4}{|c|}{$\begin{array}{l}\text { *Standardized differences were used to identify potential clinically significant differences }(>0.10) \text { between groups. } \\
\text { They are preferred over traditional hypothesis testing to assess baseline differences for population-based studies. }\end{array}$} \\
\hline \multicolumn{4}{|c|}{$\begin{array}{l}\text { tComorbidities were determined using International Classification of Diseases } 10 \text { codes and Ontario Health } \\
\text { Insurance Plan fee and diagnosis codes that were present in the previous } 2 \text { years of administrative data. }\end{array}$} \\
\hline
\end{tabular}

around the time of a COVID-19 diagnosis (Figure 1A). Surgical procedures were most commonly categorized as orthopedics/plastic surgery (Figure 1B), and hip fracture repair was the most common surgery.

Within the surgery group, 30-day postsurgical morality was $19.7 \%$ (13 of 66) for patients who underwent surgery in the 14 days before or after a positive SARS-CoV-2 swab; 30-day mortality was significantly lower in patients who had surgery in the 15-60 days after COVID-19 diagnosis $(\leq 6.2 \%[\leq 5$ of $80], p<0.05)$.

It is reassuring that only a small proportion of patients with COVID-19 required surgery. The highest number of surgical procedures was recorded around the time of COVID-19 diagnosis, likely as a result of SARS-CoV-2 testing at the time of hospital admission. There was no surge in operative cases at the end of the usual 14-day infectious period of SARS-CoV-2 to suggest that emergent surgery was delayed. Surgeons in leadership positions can use the approximate proportion of patients with COVID-19 requiring surgical interventions to help plan for future operative volumes in this group.

It is important for physicians to recognize that surgery in a patient with

\section{A}

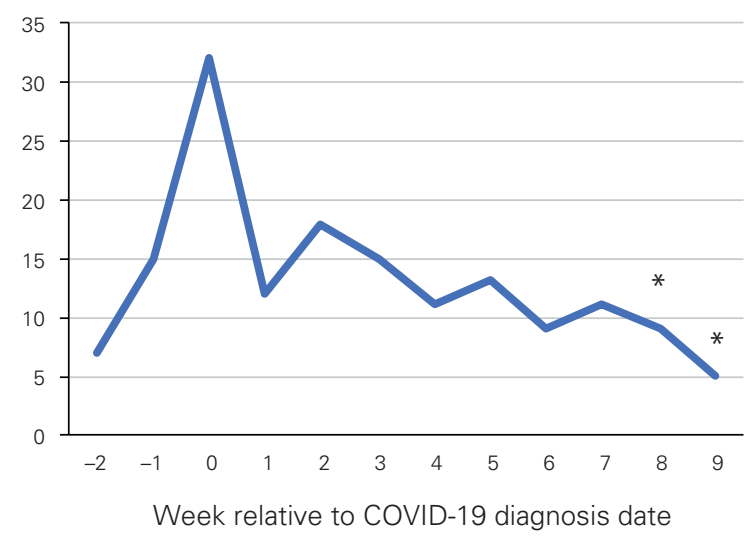

\section{B}

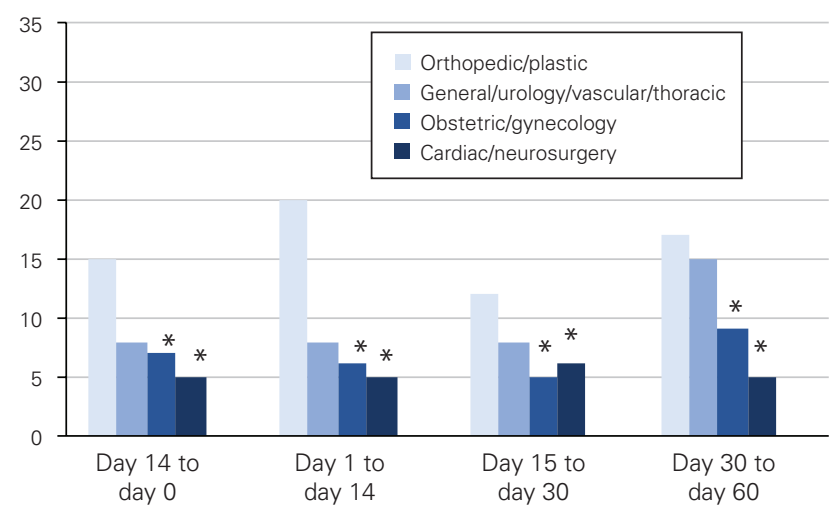

Fig. 1. Overview of surgical care provided to patients with COVID-19. (A) Number of patients requiring surgical procedures relative to their COVID-19 diagnosis. (B) Type of surgical procedure performed. Note: In keeping with privacy regulations, which prohibit the publication of small cells $(n \leq 5)$, numbers for week 8 and 9 in panel A and some of the groups in panel B are masked (indicated by an asterisk), and are less than or equal to the values shown. In panel $B, \leq 12$ of $146(\leq 8.2 \%)$ patients had procedures performed by multiple specialties on the same day and are not included in the graph. 
COVID-19 has a 1 in 5 mortality risk; however, this risk seems to be significantly attenuated 2 weeks after COVID-19 diagnosis. The 30-day mortality in our study is consistent with the $24 \%$ reported in a multinational collaboration of 1128 patients (primarily from Italy, Spain and the United Kingdom), ${ }^{2}$ and the approximately $20 \%$ from smaller Italian ${ }^{1}$ and Chinese ${ }^{3}$ case series, despite a lack of COVID-19-related health care system failures in Canada compared with some of these countries. However, our observed 30-day mortality is higher than expected when considering published rates from emergency general surgeries $(6 \%),{ }^{4}$ or after hip fracture $(7 \%) .{ }^{5}$ The high mortality during the 2 weeks before and after COVID-19 diagnosis is likely a combination COVID-19, the emergency nature of the surgery, and the increased comorbidities in patients requiring surgery.

The nature of our data did not permit more detailed information about the exact surgery and indication, or about patients' clinical status. We could not determine the cause of death or the role that COVID-19 may have played. Our small sample size and low outcome rate precluded multivariable modelling. Similar to many countries, testing capacity for SARS-CoV-2 was initially limited, therefore we may not have identified patients with mild symptoms or asymptomatic disease who had surgery.

As we enter future waves of COVID-19 in Canada, it will be important for surgeons to continue to advocate for patients who require surgery. At the same time, we must be careful to avoid surgical interventions if possible in patients who have or may have COVID-19.

Acknowledgements: This work was supported by a grant from the St. Joseph's Health Care Foundation's Health Crisis Fund. The study was supported by ICES Western. ICES is funded by an annual grant from the Ontario Ministry of Health and Long-Term Care (MOHLTC). Core funding for ICES Western is provided by the Academic Medical Organization of Southwestern Ontario (AMOSO), the
Schulich School of Medicine and Dentistry (SSMD), Western University, and the Lawson Health Research Institute (LHRI). The opinions, results and conclusions are those of the authors and are independent from the funding sources. No endorsement by ICES, AMOSO, SSMD, LHRI, or the MOHLTC is intended or should be inferred. Parts of this material are based on data and/or information compiled and provided by CIHI. However, the analyses, conclusions, opinions and statements expressed in the material are those of the author(s), and not necessarily those of CIHI.

Affiliations: From the Department of Surgery \& Epidemiology and Biostatistics, Western University, London, Ont. (Welk); ICES Western, London, Ont. (Welk, Richard); and the Department of Surgery, Humber River Hospital, Toronto, Ont. (Rodriguez-Elizalde).

Competing interests: None declared.

Contributors: All authors contributed substantially to the conception, writing and revision of this article and approved the final version for publication.

Content licence: This is an Open Access article distributed in accordance with the terms of the Creative Commons Attribution (CC BYNC-ND 4.0) licence, which permits use, distribution and reproduction in any medium, provided that the original publication is properly cited, the use is noncommercial (i.e., research or educational use), and no modifications or adaptations are made. See: https://creativecommons. org/licenses/by-nc-nd/4.0/

\section{References}

1. Doglietto F, Vezzoli M, Gheza F et al. Factors associated with surgical mortality and complications among patients with and without coronavirus disease 2019 (COVID-19) in Italy. 7AMA Surg 2020;155:691-702.

2. Nepogodiev D, Bhangu A, Glasbey JC et al. Mortality and pulmonary complications in patients undergoing surgery with perioperative SARS-CoV-2 infection: an international cohort study. Lancet 2020;396:27-38.

3. Lei S, Jiang F, Su W et al. Clinical characteristics and outcomes of patients undergoing surgeries during the incubation period of COVID-19 infection. Eclinicalmedicine 2020;21:100331.

4. Ingraham AM, Cohen ME, Raval MV, et al. Comparison of hospital performance in emergency versus elective general surgery operations at 198 hospitals. 7 Am Coll Surg 2011;212:20-28.e1.

5. Cho N, Boland L, McIsaac DI. The association of female sex with application of evidence-based practice recommendations for perioperative care in hip fracture surgery. CMA7 2019;191:E151-8. 